GLASNIK MATEMATIČKI

Vol. 40(60)(2005), $241-247$

\title{
CONVEXIFIABLE FUNCTIONS IN INTEGRAL CALCULUS
}

\author{
SANJO ZlobeC \\ McGill University, Canada
}

\begin{abstract}
A function is said to be convexifiable if it becomes convex after adding to it a strictly convex quadratic term. In this paper we extend some of the basic integral properties of convex functions to Lipschitz continuously differentiable functions on real line. In particular, we give estimates of the mean value, a "nonstandard" form of Jensen's inequality, and an explicit representation of analytic functions. It is also outlined how one can use convexification to study ordinary differential equations.
\end{abstract}

\section{IntRoduCtion}

For a given continuous $f: \mathbb{R} \rightarrow \mathbb{R}$, defined on a bounded convex set $I$ of the real line $\mathbb{R}$, consider the function $\varphi: \mathbb{R} \times \mathbb{R} \rightarrow \mathbb{R}$ defined by $\varphi(t, \alpha)=$ $f(t) \frac{1}{2} \alpha t^{2}$. If $\varphi(t, \alpha)$ is convex on $I$ for some $\alpha=\alpha^{*}$, then $\varphi(t, \alpha)$ is said to be a convexification of $f$ and $\alpha^{*}$ is its convexifier on $I$. Function $f$ is convexifiable if it has a convexification. If $\alpha^{*}$ is a convexifier of $f$, then so is every $\alpha \leq \alpha^{*}$. Indeed, $\varphi(t, \beta)=\varphi\left(t, \alpha^{*}\right)+\frac{1}{2} \beta t^{2}$ is convex for any $\beta \geq 0$, being the sum of two convex functions. Convexifiable functions have been studied on $\mathbb{R}^{n}$ in $[6,7,8]$ and characterized using the fact that for continuous functions convexity and midpoint convexity are equivalent notions [3, 4]. The class of convexifiable functions is large: Besides convex and twice continuously differentiable functions [6], it has recently been shown that this class also includes all continuously differentiable functions with Lipschitz derivative [8], i.e., loosely speaking, almost all smooth functions of practical interest.

2000 Mathematics Subject Classification. 26B25, 52A40.

Key words and phrases. Convex function, convexifiable function, integral mean value, Jensen's inequality, analytic function.

This research is sponsored in part by a grant from NSERC of Canada. 
In this paper we study only convexifiable functions of a single scalar variable. This framework enables us to use integral calculus. Two new characterizations of convexifiable functions are given in Section 2. The estimates for the integral mean-value are extended from convex function $[4,5]$ to convexifiable functions in Section 3. An integral form of Jensen's inequality for generally non-convex functions is derived in Section 4. A non-integral formulation of this inequality in $\mathbb{R}^{n}$ has been recently given in [7]. Since every analytic function is convexifiable, we obtain properties of analytic functions as special cases, in Section 5. Finally it is outlined how one can "convexify" initial-value problems in ordinary differential equation in Section 6.

\section{Scalar COnVexifiable functions}

We study continuous functions $f: \mathbb{R} \rightarrow \mathbb{R}$ on a compact interval $I=$ $[a, b], a<b$. Convexity of $f$ is defined by the behavior of $f$ at three points in $I$, say, $s \leq t \leq \xi$ where $t=\lambda s+(1-\lambda) \xi$ for some $0 \leq \lambda \leq 1$. Function $f$ is said to be convex on $I$ if at all these points

$$
f(\lambda s+(1-\lambda) \xi) \leq \lambda f(s)+(1-\lambda) f(\xi) .
$$

One can characterize convexifiable functions by the sign of a particular determinant. First, for $s<t<\xi$ denote

$X=\left(\begin{array}{c}\xi-t \\ s-\xi \\ t-s\end{array}\right), \quad B=\left(\begin{array}{c}s^{2} \\ t^{2} \\ \xi^{2}\end{array}\right), \quad \Phi=\left(\begin{array}{c}f(s) \\ f(t) \\ f(\xi)\end{array}\right), \quad V=\left(\begin{array}{ccc}1 & s & s^{2} \\ 1 & t & t^{2} \\ 1 & \xi & \xi^{2}\end{array}\right)$.

Note that $\operatorname{det} V=(s t)(t-\xi)(\xi-s)>0$.

Theorem 2.1 (Determinant Characterization of Convexifiable Function). Function $f: I \rightarrow \mathbb{R}$ is convexifiable if, and only if, there exists a number $\alpha$ such that

$$
\operatorname{det}\left(\begin{array}{ccc}
1 & 1 & 1 \\
s & t & \xi \\
f(s) & f(t) & f(\xi)
\end{array}\right) \geq \frac{1}{2} \alpha \operatorname{det} V
$$

for every three points $s<t<\xi$ in $I$.

Proof. This result with $\alpha=0$ is a characterization of convex function; e.g., $[2,4,5]$. When applied to the convexification, it yields (1).

For three important classes of functions, a convexifier $\alpha$ can be given explicitly. If $f$ is convex then one can specify $\alpha=0$. If $f$ is twice continuously differentiable on $I$, then a convexifier is $\alpha=\lambda^{*}=\min _{t \in I} f^{\prime \prime}(t)$, e.g., [6]. If $f$ is Lipschitz continuously differentiable, i.e., $\left|f^{\prime}(s)-f^{\prime}(t)\right| \leq L|s-t|$ for every $s, t \in I$ and some constant $L$, then $\alpha=-L$ is a convexifier, e.g., [8]. Convexifiable functions can be represented explicitly: 
Theorem 2.2 (Explicit Representation of Convexifiable Function). Function $f: I \rightarrow \mathbb{R}$ is convexifiable if, and only if, there exists a number $\alpha$ such that

$$
f(t)=f(c)+\frac{1}{2} \alpha\left(t^{2} c^{2}\right)+\int_{c}^{t} g(\xi) d \xi
$$

where $c, t \in I, c<t$, and $g=g(\cdot, \alpha): I \rightarrow \mathbb{R}$ is a non-decreasing rightcontinuous function.

Proof. Inequality (2) with $\alpha=0$ is a characterization of convex function, e.g., [5, Theorem 1.13]. If $f$ is convexifiable then there is an $\alpha$ such that $\varphi(t, \alpha)$ is convex in $t$. Hence (2) holds for this $\alpha$. On the other hand, if (2) holds then, after back substitution, $\varphi(t, \alpha)$ is a convexification.

EXAMPLE 2.3. Let us check whether, e.g., $f(t)=-|t|$ is convexifiable on $I=[-1,1]$. Inequality (1), at $s=-\epsilon, t=0, \xi=\epsilon, \epsilon>0$ yields $\alpha \leq-2 / \epsilon$, which is unbounded from below as $\epsilon \rightarrow 0$. Hence $f$ is not convexifiable. This shows that convexification by quadratic is not suitable for non-smooth functions. On the other hand, take, e.g., $f(t)=\cos k t$. This $f$ is locally strictly concave around $t=0$. But it is convexifiable on $t \in I=[-\pi, \pi]$ with convexifier $\alpha=-k^{2}$.

Example 2.4 (Bound on Fourier Coefficients). If $f:[0,2 \pi] \rightarrow \mathbb{R}$ is continuous and convexifiable with a convexifier $\alpha$, then for $k \in\{1,2, \ldots\}$ we estimate the Fourier coefficient:

$$
a_{k}=\frac{1}{\pi} \int_{0}^{2 \pi} f(t) \cos k t d t \geq 2 \frac{\alpha}{k^{2}}
$$

Indeed, if $f$ were convex, then one could set $\alpha=0$ and hence it follows that $a_{k} \geq 0$ by, e.g., [4, p.8; Problem G]. We obtain the new inequality after substituting $f$ by its convexification $\varphi(t, \alpha)$ and integration.

\section{INTEGRAL MEAN VALUe}

One of the fundamental results of integral calculus is the mean value theorem. It says that for a continuous function $f$ on $I=[a, b], a<b$, there exists $\xi \in[a, b]$ such that

$$
f(\xi)=\frac{1}{b-a} \int_{a}^{b} f(t) d t
$$

The number $f(\xi)$ is interpreted as the mean (average) value of $f$ on the interval $[a, b]$. If $f:(a, b) \rightarrow \mathbb{R}$ is a convex function then it is well known that the mid-point value of $f$ can not be higher than its mean value, i.e.

$$
f((a+b) / 2) \leq \frac{1}{b-a} \int_{a}^{b} f(t) d t .
$$

An extension from convex to convexifiable function follows: 
Theorem 3.1 (Mean-Value Bound for Convexifiable Function; [8]). Consider a continuous convexifiable function $f: \mathbb{R} \rightarrow \mathbb{R}$ with a convexifier $\alpha$ on an open interval $(a, b) \in \mathbb{R}$. Then

$$
\int_{s}^{t} \frac{f(\xi)}{t-s} d \xi \leq \frac{1}{2}[f(s)+f(t)] \frac{1}{12} \alpha(t s)^{2}
$$

for every $a<s<t<b$.

A composite version of this result follows. First we recall it for a convex function:

Theorem 3.2 (Integral Mean-Value Bound for Convex Composite Function [5]). Let $f:(a, b) \rightarrow \mathbb{R}$ be a convex function and let $g:[c, d] \rightarrow(a, b)$ be continuous. Then

$$
f\left(\frac{1}{d-c} \int_{c}^{d} g(t) d t\right) \leq \frac{1}{d-c} \int_{c}^{d} f(g(t)) d t .
$$

If $g(t)=t$, then (4) recovers (3). One may replace $g$ by a function which is only integrable in the Lebesque sense over $[c, d]$. This estimate is important in probability theory: If $X$ is a probability space, with probability measure $\mu$ (so that $\mu(X)=1$ ) and if $f:(a, b) \rightarrow \mathbb{R}$ is $\mu$-integrable, then

$$
f\left(\int_{X} g d \mu\right) \leq \int_{X}(f \circ g) d \mu .
$$

In probabilistic terms: if $x$ is a random variable on $X$, then $f(E x) \leq E[(f(x)]$, where $E x$ is the expectation of $x$. The above results can be extended to composite convexifiable functions:

Theorem 3.3 (Integral Mean-Value Bound for Composite Convexifiable Function). Let $f:(a, b) \rightarrow \mathbb{R}$ be convexifiable with a convexifier $\alpha$ and let $g:[c, d] \rightarrow(a, b)$ be continuous. Then

$$
f\left(\frac{1}{d-c} \int_{c}^{d} g(t) d t\right) \leq \frac{1}{d c} \int_{c}^{d} f(g(t)) d t+\frac{1}{2} \alpha R(c, d ; g)
$$

where

$$
R(c, d ; g)=\left[\frac{1}{d-c} \int_{c}^{d} g(t) d t\right]^{2}-\frac{1}{d-c} \int_{c}^{d}[g(t)]^{2} d t .
$$

Proof. Since $f$ is convexifiable, $\varphi(t, \alpha)=f(t) \frac{1}{2} \alpha t^{2}$ is convex in $t$ for any convexifier $\alpha$. Hence (4) applies. After manipulation with integrals one obtains (5).

Remark 3.4 (Special Cases). Let $f:(a, b) \rightarrow \mathbb{R}$ and $g:[c, d] \rightarrow(a, b)$ be continuous on $(a, b)$. Then in Theorem 3.3 one can specify $\alpha=0$, if $f$ is convex on $(a, b)$ (This is (4)). Also $\alpha=\lambda^{*}=\min _{t \in I} f^{\prime \prime}(t)$, if $f$ is 
twice continuously differentiable or analytic on $(a, b)$, and $\alpha=-L$, if $f$ is continuously differentiable with Lipschitz derivative and some constant $L$ on $(a, b)$.

\section{Integral Jensen's inequality}

Jensen's inequality has been formulated and studied in the literature for convex functions. Recently a vector-space version has been given for nonconvex functions in [7]. Here we give an integral formulation of this "nonconvex result".

Theorem 4.1 (Integral Jensen's Inequality for Convexifiable Function). Let $f:(a, b) \rightarrow \mathbb{R}$ be convexifiable with a convexifier $\alpha$ and let $g:[c, d] \rightarrow \mathbb{R}$ be integrable with $a<g(t)<b$. If $\lambda:[c, d] \rightarrow \mathbb{R}$ is non-negative, if both

$$
\int_{c}^{d} \lambda(t) d t=1, \text { and } \lambda g \text { is integrable on }[c, d]
$$

then

$$
\left.f\left(\int_{c}^{d} \lambda(t) g(t)\right) d t\right) \leq \int_{c}^{d} \lambda(t) f(g(t)) d t+\frac{1}{2} \alpha J(c, d ; g),
$$

where

$$
J(c, d ; g)=\left[\int_{c}^{d} \lambda(t) g(t) d t\right]^{2}-\int_{c}^{d} \lambda(t)[g(t)]^{2} d t .
$$

Proof. The inequality for convex functions (case: $\alpha=0$ ) is given in, e.g., $\left[4\right.$, p. 193]. We apply this result to the convexification $\varphi(t, \alpha)=f(t) \frac{1}{2} \alpha t^{2}$.

口

REMark 4.2. "Correctifiers" $R(c, d ; g)$ and $J(c, d ; g)$ do not depend on the function $f$.

\section{AnALYtic FUnCtions}

Since every analytic function is twice continuously differentiable, it is convexifiable and one can specify, e.g., $\lambda^{*}=\min _{t \in I} f^{\prime \prime}(t)$ as a convexifier, e.g., [8]. The above results are readily applicable. Some of the implications are listed below.

Corollary 5.1. If $f: I \rightarrow \mathbb{R}$ is analytic, then for every $\alpha \leq \lambda^{*}$, (1) holds at every three points $s<t<\xi$ in $I$.

Corollary 5.2 (Explicit Representation of Analytic Function). If $f$ : $I \rightarrow \mathbb{R}$ is analytic, then, for every $\alpha \leq \lambda^{*}, f$ is of the form (2), where $c, t \in I$ and $g=g(\cdot, \alpha): I \rightarrow \mathbb{R}$ is a non-decreasing right-continuous function.

Being convexifiable, an arbitrary analytic function, when considered on a compact interval, is the difference of an analytic convex function and a strictly convex quadratic. 


\section{Convexification of Differential EQUATIONS}

Using the idea of convexification one can study ordinary differential equations. Assuming that solution is a convexifiable function one can look for it in the form $y(t)=\varphi(t, \alpha)+\frac{1}{2} \alpha t^{2}$ where $\alpha$ is a convexifier. After substitution for $y$, the differential equation in $y$ becomes a differential equation in $\varphi$. The novelty here is that the new transformed differential equation has a convex solution. Since the theory of convex functions is well studied, this approach may have theoretical and numerical advantages over the usual approaches. The convexification applies also to the initial conditions.

EXAMPLE 6.1 (Convexification of ODE). Consider the initial value problem

$$
\frac{d y}{d t}+y=0, \quad y(0)=-2 .
$$

The solution is a concave function $y=-2 e^{t}$. It is also obtained after convexification: substitution for $y=\varphi+\frac{1}{2} \alpha t^{2}$ yields the "convexified" problem

$$
\frac{d \varphi}{d t}+\varphi=-\alpha\left(t+\frac{1}{2} t^{2}\right), \quad \varphi(0)=-2 .
$$

Its solution, with some convexifier $\alpha$, is $\varphi=-2 e^{-t}-\frac{1}{2} \alpha t^{2}$. Back substitution yields the answer to the original problem.

Since all Lipschitz continuously differentiable functions are convexifiable, this approach is applicable, loosely speaking, to almost all initial value problems describing real-life situations. In contrast, related results in the literature typically deal with the behavior of the entire set of solutions under the actions of various transformation groups, not with individual solutions.

ACKNOWLEDGEMENTS.

The author is indebted to Professor N. Kamran for his comments related to Section 6.

\section{REFERENCES}

[1] J. L. W. V. Jensen, Sur les fonctions convexes et les inegalites entre les valeurs moyennes, Acta Math. 30 (1906) 175-193.

[2] S. Karlin and Z. Ziegler, Some applications to inequalities of the method of generalized convexity, J. d' Analyse Math. 30 (1976) 281-303.

[3] S. Kurepa, Convex functions, Glasnik Mat. Fiz. Astron. II 11 (1956) 89-93.

[4] A.W. Roberts and D. E. Varberg, Convex Functions, Academic Press, 1973.

[5] J. van Tiel, Convex Analysis, Wiley, Chichester, 1984.

[6] S. Zlobec, Estimating convexifiers in continuous optimization, Math. Comm. 8 (2003) 129-137.

[7] S. Zlobec, Jensen's inequality for non-convex functions, Math. Comm. 9 (2004) 119124.

[8] S. Zlobec, Characterization of convexifiable functions, McGill University, June 2004, preprint. 
S. Zlobec

McGill University

Department of Mathematics and Statistics

Burnside Hall, 805 Sherbrooke Street West

Montreal, Quebec, Canada H3A 2K6

E-mail: zlobec@math.mcgill.ca

Received: 19.11.2004. 